\title{
Two-dimensional structure of thin transonic discs: Theory and observational manifestations
}

\author{
V. Beskin ${ }^{1}$ and A. Tchekhovskoy ${ }^{2}$ \\ ${ }^{1}$ Lebedev Physical Institute, Leninskii prosp. 53, Moscow 119991, Russia \\ e-mail: beskin@lpi.ru \\ 2 Moscow Institute of Physics and Technology, Institutskii per. 9, Dolgoprudny 141700, Russia \\ e-mail: chekhovs@lpi.ru
}

Received 4 July 2004 / Accepted 23 November 2004

\begin{abstract}
We study the two-dimensional structure of thin transonic accretion discs in the vicinity of black holes. We use the hydrodynamical version of the Grad-Shafranov equation and focus on the region inside the marginally stable orbit (MSO), $r<r_{\mathrm{ms}}$. We show that all components of the dynamical force in the disc become significant near the sonic surface and especially important in the supersonic region. Under certain conditions, the disc structure is shown to be far from radial, and we review the affected disc properties, in particular the role of the critical condition at the sonic surface: it determines neither the accretion rate nor the angular momentum in the accretion disc. Finally, we present a simple model explaining quasi-periodical oscillations that have been observed in the infrared and X-ray radiation of the Galactic Centre.
\end{abstract}

Key words. hydrodynamics - accretion, accretion disks - black hole physics

\section{Introduction}

The investigation of accretion flows near black holes (BHs) is undoubtedly of great astrophysical interest. Substantial energy is released near BHs, and general relativity effects, attributable to strong gravitational fields, appear there. Depending on external conditions, both quasi-spherical and disc accretion flows can be realized.

The studies of thin disc accretion span more than three decades. Many results were included in textbooks (Shapiro \& Teukolsky 1983; Lipunov 1992). Lynden-Bell (1969) was the first to point out that supermassive BHs surrounded by accretion discs could exist in galactic nuclei. Subsequently, a theory for such discs was developed that is now called the standard model, or the model of the $\alpha$-disc (Shakura 1972; Shakura \& Sunyaev 1973; Novikov \& Thorne 1973).

According to the standard model, if the accreting gas temperature is low enough for the speed of sound $c_{\mathrm{s}}$ to be much lower than the Keplerian rotation velocity $v_{\mathrm{K}}$, matter forms a thin equilibrium disc and moves in nearly circular orbits with the Keplerian velocity $v_{\mathrm{K}}(r)=(G M / r)^{1 / 2}$, where $M$ is the black hole mass and $G$ is the gravitational constant. The disc thickness within this model is determined by the balance of gravitational and accreting matter pressure forces,

$H \approx r \frac{c_{\mathrm{s}}}{v_{\mathrm{K}}}$.

Minor friction between rotating gas layers leads to the loss of angular momentum, so that the accreting matter gradually approaches the compact object; this motion only slightly disturbs the vertical balance ${ }^{1}$ and is commonly considered a small perturbation. To account for this phenomenon, the standard model introduces a phenomenological proportionality coefficient $\alpha_{\mathrm{SS}} \leq 1$ between the viscous stress tensor $t_{\varphi}^{r}$ and the pressure $P, t_{\varphi}^{r}=\alpha_{\mathrm{SS}} P$. As a result, the radial velocity $v_{r}$ can be evaluated as

$\frac{v_{r}}{v_{\mathrm{K}}} \approx \alpha_{\mathrm{SS}} \frac{c_{\mathrm{s}}^{2}}{v_{\mathrm{K}}^{2}}$,

so that for $c_{\mathrm{s}} \ll v_{\mathrm{K}}$ the radial velocity is much lower than both the Keplerian velocity and the speed of sound.

The foregoing is valid far from the compact object where the relativistic effects are unimportant. As for the inner regions of the accretion disc, the general relativity effects lead to at least two new qualitative phenomena. First, there are no stable circular orbits at small radii, $r<r_{\mathrm{ms}}$ for a non-rotating $\mathrm{BH}$; $r_{\mathrm{ms}}=3 r_{\mathrm{g}}$ is the radius of the marginally stable orbit (MSO) and $r_{\mathrm{g}}=2 G M / c^{2}$ is the $\mathrm{BH}$ gravitational radius. This means that the accreting matter that falls into the region $r<r_{\text {ms }}$ rapidly approaches the $\mathrm{BH}$ horizon ${ }^{2}$ regardless of viscosity. Second, thin disc accretion on to BHs is always transonic. This results from the fact that, according to (2), the flow is subsonic outside

${ }^{1}$ Let the vertical, or transversal, direction be a $\theta$-direction in the spherical system of coordinates with the half-line $\theta=0$ being aligned with the angular momentum vector of the infalling matter. Then, the longitudinal direction is a radial one.

${ }^{2}$ In the dynamic time $\tau_{\mathrm{d}} \sim\left[v_{r}\left(r_{\mathrm{ms}}\right) / c\right]^{-1 / 3} r_{\mathrm{g}} / c$. 
the MSO, $r>r_{\mathrm{ms}}$, while at the horizon $r=r_{\mathrm{g}}$ the flow is supersonic (Beskin 1997). Therefore, the study of inner accretion disc regions requires a consistent analysis of transonic flows.

Transonic accretion on to BHs has been the subject of many research publications. Of crucial importance was the paper by Paczyński \& Bisnovatyi-Kogan (1981) who formulated equations of motion in terms of radial derivatives only: they used quantities averaged over the disc thickness. Subsequently, many authors considered only such one-dimensional models. In most cases, the model potential of Paczyński \& Wiita (1980), $\varphi_{\mathrm{g}}=-G M /\left(r-r_{\mathrm{g}}\right)$, which simulates the properties of the strong gravitational field of a centrally symmetric BH, was used (Abramowicz et al. 1988; Papaloizou \& Szuszkiewicz 1994; Chen et al. 1997; Narayan et al. 1997; Artemova et al. 2001). The Schwarzschild and Kerr metrics that describe the gravitational field of axisymmetric BHs were far more rare (Riffert \& Herold 1995; Peitz \& Appl 1997; Beloborodov 1998; Gammie \& Popham 1998a,b). As for the two- and three-dimensional structure, it was investigated mostly only numerically and only for thick discs (Papaloizou \& Szuszkiewicz 1994; Igumenshchev \& Beloborodov 1997; Balbus \& Hawley 1998; Igumenshchev et al. 2000; Krolik \& Hawley 2002; Igumenshchev et al. 2003; De Villiers et al. 2003; Gammie et al. 2004).

Therefore, for thin accretion discs basic results were obtained in terms of the one-dimensional approach. However, the procedure for averaging over the disc thickness, which is likely to be valid in the region of stable orbits, in fact requires a more serious analysis. In our view, it is the assumption that the transverse velocity $v_{\theta}$ may always be neglected in thin accretion discs, i.e. that the disc thickness is always determined by Eq. (1), that is the most debatable. This assumption is widely used, explicitly or implicitly, virtually in all papers devoted to thin accretion discs (see the papers cited above; and Abramowicz \& Zurek 1981; Chakrabarti 1996).

Abramowicz et al. (1997) pointed out the importance of allowing for the transverse velocity in the inner accretion disc regions. They argued that the transverse component of the dynamical force has the same asymptotic near the equatorial plane as the transversal components of the gravitational and pressure forces (under the natural assumption $v_{\theta} \propto \cos \theta$ in the limit $\cos \theta \rightarrow 0$ ). Therefore, the fact that the transversal velocity vanishes on the equator by no means implies that $[(\boldsymbol{v} \nabla) \boldsymbol{v}]_{\theta}$ may be ignored. However, the authors concluded that for thin discs the dynamical term may still be disregarded so that the accretion disc thickness can be determined from the balance of the gravitational force and the pressure gradient up to the $\mathrm{BH}$ horizon. We show that this conclusion is inapplicable for the class of problems we consider below.

To refine the issue, recall the simplest case of transonic accretion, namely ideal spherically symmetric accretion (Bondi 1952). Far outside the sonic surface in such a flow the dynamical term is negligible in comparison to the pressure gradient and the gravitational force. However, all the terms become of the same order of magnitude near the sonic surface. Moreover, after passing the sonic surface, the motion of matter differs little from free-fall, therefore the pressure gradient no longer affects the dynamics of accreting matter. This makes it seem unreasonable to determine the disc thickness from Eq. (1), at least in the supersonic region.

However, Eq. (1) can be easily modified to account for dynamical forces. Indeed, we have at the border of the disc

$v_{\Theta} \approx \frac{\mathrm{d} H}{\mathrm{~d} r} v_{r}$

where $\Theta=\pi / 2-\theta$. Now assuming linear dependence of $v_{\Theta}$ on $\Theta$ for $\Theta \ll 1$, we obtain

$v_{\Theta} \approx\left(\frac{r}{H} \frac{\mathrm{d} H}{\mathrm{~d} r}\right) v_{r} \Theta$

With this in hand the dynamical term can be written as

$$
\begin{aligned}
{[(\boldsymbol{v} \nabla) v]_{\Theta}=} & v_{r} \frac{\partial\left(r v_{\Theta}\right)}{r \partial r}+v_{\Theta} \frac{\partial v_{\Theta}}{r \partial \Theta} \\
\approx & \frac{v_{r}}{r} \frac{\partial}{\partial r}\left(r \frac{r}{H} \frac{\mathrm{d} H}{\mathrm{~d} r} v_{r} \Theta\right) \\
& +\left(\frac{r}{H} \frac{\mathrm{d} H}{\mathrm{~d} r} v_{r} \Theta\right) \frac{\partial}{r \partial \Theta}\left(\frac{r}{H} \frac{\mathrm{d} H}{\mathrm{~d} r} v_{r} \Theta\right) .
\end{aligned}
$$

Now, assuming $\Theta=H / r$ and using Eq. (5), the $\Theta$-component of the Euler equation, divided by $\left(-\rho H / r^{2}\right)$, can be written as

$r v_{r} \frac{\mathrm{d}}{\mathrm{d} r}\left[\left(\frac{r}{H} \frac{\mathrm{d} H}{\mathrm{~d} r}\right) v_{r}\right]+\left(\frac{r}{H} \frac{\mathrm{d} H}{\mathrm{~d} r}\right)^{2} v_{r}^{2}=c_{\mathrm{s}}^{2} \frac{r^{2}}{H^{2}}-v_{\varphi}^{2}$,

where the two terms on the 1.h.s. correspond to the dynamical force components $v_{r} \partial\left(r v_{\theta}\right) /(r \partial r)$ and $v_{\theta} \partial v_{\theta} /(r \partial \theta)$ respectively. The r.h.s. of Eq. (6) corresponds to the discrepancy between the centrifugal force $v_{\varphi}^{2} \tan \Theta / r$ and the pressure force $\partial P /(r \partial \Theta)$ whose absolute value is evaluated as $P / H \sim \rho c_{\mathrm{s}}^{2} / H$. This discrepancy was postulated to vanish in Eq. (1).

Accounting for the dynamical forces, Eq. (6) is a simple qualitative analogue of the second-order Grad-Shafranov Eq. (14) and allows significant disc thickness deviations from the value given by conventional prescription (1). For instance, nondimensionalizing Eq. (6) yields $\delta r$, the characteristic radial scale of change of $H$ near the sonic surface $r=r_{*}$, $\delta r\left(r_{*}\right) \sim H\left(r_{*}\right)$. Consistently, by Eq. (3) it follows that $v_{\theta} \sim v_{r}$ which indeed indicates a rapid change of the disc thickness. Naturally, in the region of stable orbits in a similar way we get $\delta r\left(r>r_{\text {ms }}\right) \sim r$ and $v_{\theta} \sim v_{r} H / r \ll v_{r}$ which justifies the use of conventional prescription (1) outside the MSO.

The existence of the small longitudinal scale $\delta r\left(r_{*}\right) \ll r_{\mathrm{g}}$ is one of the key results of this paper. Note also that since Eq. (6) is a second-order differential equation for $H$, there in fact exist two additional degrees of freedom, frequently overlooked, and only one of the two is fixed by the critical condition at the sonic surface. This hints that the critical condition determines neither the accretion rate nor the angular momentum in the disc but only imposes a restriction on the function $H(r)$, i.e. on the form of the flow, near the sonic surface.

In this paper we purposefully consider extreme boundary conditions with radial inflow velocity at the MSO being far smaller than the speed of sound. This allows us to perform the study of the problem analytically. Moreover, the results turn out to be almost independent of the radial inflow velocity, and 
the observational predictions of our model depend only on the the black hole mass and its angular momentum and are totally independent of the inflow velocity and the speed of sound. This model offers an insight into the fundamental properties we might lose if we do not allow for the transversal velocity in thin accretion discs. This is the first step in understanding the physics of the critical condition at the sonic surface and its influence on the global disc structure.

In the next section we introduce the reader to the hydrodynamical version of the Grad-Shafranov equation in the Schwarzschild metric and derive some useful relations. In Sects. 3 and 4 we sequentially study the subsonic and the sonic surface regions. Section 5 presents the study of the supersonic flow for the case of a spinning BH, i.e. Kerr metric, followed by the discussion of its applications to the explanation of quasiperiodical oscillations detected in the infrared and X-ray observations of the Galactic Centre (Sect. 6).

\section{Basic equations}

We consider thin disc accretion on to a $\mathrm{BH}$ in the region where there are no stable circular orbits. As argued above, the contribution of viscosity should no longer be significant here. Hence we may assume that an ideal hydrodynamics approach is suitable to describe the flow structure in this inner area of the accretion disc. Below, unless specifically stated, we consider the case of a non-spinning BH, i.e. use the Schwarzschild metric, and use a system of units with $c=G=1$. We measure radial distances in units of $M$, the $\mathrm{BH}$ mass.

In Boyer-Lindquist coordinates the Schwarzschild metric is (Landau \& Lifshits 1987a)

$\mathrm{d} s^{2}=-\alpha^{2} \mathrm{~d} t^{2}+g_{i k} \mathrm{~d} x^{i} \mathrm{~d} x^{k}$,

where

$\alpha^{2}=1-2 / r, \quad g_{r r}=\alpha^{-2}, \quad g_{\theta \theta}=r^{2}, g_{\varphi \varphi}=\varpi^{2}=r^{2} \sin ^{2} \theta$.

Here and below indices without caps denote vector components with respect to the coordinate basis in "absolute" threedimensional space, and indices with caps denote their physical components. The $\nabla_{k}$ symbol always represents covariant derivative in "absolute" three-dimensional space with $g_{i k}$ metrics (8).

We reduce our discussion to the case of axisymmetric stationary flows. This makes it possible to introduce a stream function $\Phi(r, \theta)$ which defines the physical poloidal 4-velocity component $\boldsymbol{u}_{\hat{\mathrm{p}}} \equiv u_{\hat{r}} \boldsymbol{e}_{\hat{r}}+u_{\hat{\theta}} \boldsymbol{e}_{\hat{\theta}}$ as

$\alpha n \boldsymbol{u}_{\hat{\mathrm{p}}}=\frac{1}{2 \pi \varpi}\left(\boldsymbol{\nabla} \Phi \times \boldsymbol{e}_{\hat{\varphi}}\right)$,

where $n$ is the particle concentration in the comoving reference frame. The surfaces $\Phi(r, \theta)=$ const determine the streamlines of the matter ( $\Phi=0$ corresponds to the streamline $\theta=0$ ).

For an ideal flow there are three integrals of motion conserved along the streamlines, namely entropy,

$S=S(\Phi)$

energy flux,

$E(\Phi)=\mu \alpha \gamma$, and the $z$-component of angular momentum,

$L(\Phi)=\mu \varpi u_{\hat{\varphi}}$

where $\mu=\left(\rho_{m}+P\right) / n$ is relativistic enthalpy; $\rho_{m}$ is internal energy density, $P=n T$ is pressure.

As a result the relativistic Euler equation (Frolov \& Novikov 1998)

$n u^{b} \nabla_{b}\left(\mu u_{a}\right)+\nabla_{a} P-\mu n\left(u_{\hat{\varphi}}\right)^{2} \frac{1}{\varpi} \nabla_{a} \varpi+\mu n \gamma^{2} \frac{1}{\alpha} \nabla_{a} \alpha=0$,

in which indices $a$ and $b$ take on $r$ and $\theta$ values, can be rewritten in the form of the Grad-Shafranov scalar equation for $\Phi(r, \theta)$ (Beskin 1997),

$$
\begin{aligned}
-\mathcal{M}^{2}[ & \left.\frac{1}{\alpha} \nabla_{k}\left(\frac{1}{\alpha \varpi^{2}} \nabla^{k} \Phi\right)+\frac{1}{\alpha^{2} \varpi^{2}(\nabla \Phi)^{2}} \frac{\nabla^{i} \Phi \nabla^{k} \Phi \nabla_{i} \nabla_{k} \Phi}{D}\right] \\
& +\frac{\mathcal{M}^{2} \nabla_{k}^{\prime} F \nabla^{k} \Phi}{2 \alpha^{2} \varpi^{2}(\nabla \Phi)^{2} D}+\frac{64 \pi^{4}}{\alpha^{2} \varpi^{2} \mathcal{M}^{2}} \\
& \times\left(\varpi^{2} E \frac{\mathrm{d} E}{\mathrm{~d} \Phi}-\alpha^{2} L \frac{\mathrm{d} L}{\mathrm{~d} \Phi}\right)-16 \pi^{3} n T \frac{\mathrm{d} S}{\mathrm{~d} \Phi}=0,
\end{aligned}
$$

where the $\nabla_{k}^{\prime}$ derivative acts on all the variables except the quantity $\mathcal{M}$. The equation above contains three integrals $E(\Phi)$, $L(\Phi)$, and $S(\Phi)$. Here

$D=-1+\frac{1}{u_{\mathrm{p}}^{2}} \frac{c_{s}^{2}}{1-c_{s}^{2}}$,

$F=\frac{64 \pi^{4}}{\mathcal{M}^{4}}\left[\varpi^{2} E^{2}-\alpha^{2} L^{2}-\varpi^{2} \alpha^{2} \mu^{2}\right]$

and the thermodynamical function $\mathcal{M}^{2}$ is defined as

$\mathcal{M}^{2}=\frac{4 \pi \mu}{n}$.

Equation (14) contains the only singular surface - the sonic surface which is defined by the condition $D=0$. At this surface the equation changes its type from elliptical to hyperbolic.

To close the system, we need to supply the Grad-Shafranov equation with the relativistic Bernoulli equation, $u_{\hat{\mathrm{p}}}^{2}=\gamma^{2}-u_{\hat{\varphi}}^{2}-1$, which now becomes

$u_{\hat{\mathrm{p}}}^{2}=\frac{E^{2}-\alpha^{2} L^{2} / \varpi^{2}-\alpha^{2} \mu^{2}}{\alpha^{2} \mu^{2}}$.

After using definition (9) we reach

$E^{2}=\alpha^{2} \mu^{2}+\frac{\alpha^{2}}{\varpi^{2}} L^{2}+\frac{\mathcal{M}^{4}}{64 \pi^{4} \varpi^{2}}(\nabla \Phi)^{2}$.

For simplification we adopt the polytropic equation of state $P=$ $k(S) n^{\Gamma}$ so that temperature and sound velocity can be written as (Shapiro \& Teukolsky 1983)

$T=k(S) n^{\Gamma-1} ; \quad c_{\mathrm{s}}^{2}=\frac{\Gamma}{\mu} k(S) n^{\Gamma-1}$.

Since the disc is thin, $c_{\mathrm{s}} \ll 1$ (see Eq. (1)). Therefore we can write $\mu=m_{\mathrm{p}}+m_{\mathrm{p}} W$, where $W=c_{\mathrm{s}}^{2} /(\Gamma-1)$ is non-relativistic enthalpy and $m_{\mathrm{p}}$ is particle mass. For an ideal gas with $\Gamma=$ const. the function $k(S)$ can be shown to have a quite definite form

$k(S)=k_{0} \exp [(\Gamma-1) S]$ 
which for the case of the polytropic equation of state can be obtained from Eq. (20) and the thermodynamical relationship $\mathrm{d} P=n \mathrm{~d} \mu-n T \mathrm{~d} S$.

Note that Eq. (19) enables us to express the quantity $\mathcal{M}$ and together with it all the other thermodynamical quantities in terms of the potential $\Phi(r, \theta)$ and the three motion invariants. Consequently, Eq. (14) contains the only unknown function $\Phi(r, \theta)$.

\section{Subsonic flow}

In this section we show that the role of the dynamical terms becomes dominant closer to the sonic surface $r=r_{*}(\theta)$. The problem of passing through the sonic surface and the supersonic flow structure are dealt with later in this paper. Here we limit our discussion to the subsonic region only, where the poloidal velocity $u_{\hat{\mathrm{p}}}$ is far lower than the sound velocity $c_{\mathrm{s}}$ (as we assume in Sect. 1 , the condition $u_{\hat{\mathrm{p}}}^{2} / c_{\mathrm{s}}^{2} \ll 1$ holds at the MSO, and in this section we always neglect the terms of the order of $u_{\hat{\mathrm{p}}}^{2} / c_{\mathrm{s}}^{2}$ or higher). We can simplify the Grad-Shafranov hydrodynamic Eq. (14) in this limit by neglecting terms proportional to $D^{-1}$ :

$-\mathcal{M}^{2} \frac{1}{\alpha} \nabla_{k}\left(\frac{1}{\alpha \varpi^{2}} \nabla^{k} \Phi\right)+\frac{64 \pi^{4}}{\alpha^{2} \varpi^{2} \mathcal{M}^{2}}$

$\times\left(\varpi^{2} E \frac{\mathrm{d} E}{\mathrm{~d} \Phi}-\alpha^{2} L \frac{\mathrm{d} L}{\mathrm{~d} \Phi}\right)-16 \pi^{3} n T \frac{\mathrm{d} S}{\mathrm{~d} \Phi}=0$.

The resulting equation describes the subsonic flow and is elliptical. Hence, it contains no critical surfaces, and our problem requires five boundary conditions: three conditions determine the integrals of motion and the two remaining ones are the boundary conditions for the second-order Grad-Shafranov equation.

Following Sect. 1, we assume that the $\alpha$-disc theory holds outside the MSO, and we use its predictions for velocity components of the infalling matter at the $\mathrm{MSO}^{3}$ as the first three boundary conditions for our problem. For the sake of simplicity we consider the radial velocity, which is responsible for the inflow, to be constant at the surface $r=r_{\mathrm{ms}}$ and equal to $u_{0}$ and the toroidal velocity to be exactly equal to that of a free particle revolving at $r=r_{\mathrm{ms}}{ }^{4}$ :

$$
\begin{gathered}
u_{\hat{r}}\left(r_{\mathrm{ms}}, \Theta\right)=-u_{0}, \\
u_{\hat{\Theta}}\left(r_{\mathrm{ms}}, \Theta\right)=\Theta u_{0}, \\
u_{\hat{\varphi}}\left(r_{\mathrm{ms}}, \Theta\right)=1 / \sqrt{3},
\end{gathered}
$$

where $\left|u_{\hat{\Theta}}\left(r_{\mathrm{ms}}, \Theta\right)\right| \ll\left|u_{\hat{r}}\left(r_{\mathrm{ms}}, \Theta\right)\right|$ accounts for the fact that the flow is parallel ${ }^{5}$ at the MSO. The angular variable $\Theta=\pi / 2-\theta$ is counted off from the equator in the vertical direction. For the sake of convenience, we also introduce another angular variable $\Theta_{\mathrm{ms}}=\Theta_{\mathrm{ms}}(\Phi(r, \Theta))$, a Lagrange coordinate of streamlines. This is a function of streamlines. For each streamline,

\footnotetext{
${ }^{3}$ A nearly parallel inflow with small radial velocity $v_{r}$ Eq. (2).

${ }^{4}$ For a free particle revolving at $r=r_{\mathrm{ms}}$ around a non-spinning BH we have $u_{\hat{\varphi}}\left(r_{\mathrm{ms}}\right)=1 / \sqrt{3}, \alpha_{0}=\alpha\left(r_{\mathrm{ms}}\right)=\sqrt{2 / 3}, \gamma_{0}=\gamma\left(r_{\mathrm{ms}}\right)=\sqrt{4 / 3}$ (Landau \& Lifshits 1987a).

${ }^{5}$ Note that the parallel flow is not exactly the same as the radial one.
}

it gives the streamline's $\Theta$-coordinate at the MSO $r=r_{\mathrm{ms}}$. In other words, both points $(r, \Theta)$ and $\left(r_{\mathrm{ms}}, \Theta_{\mathrm{ms}}\right)$ belong to the same streamline. Further, noting that $\Theta_{\mathrm{ms}}\left(r_{\mathrm{ms}}, \Theta\right) \equiv \Theta$ and using Eqs. (9) and (23), we arrive at

$\mathrm{d} \Phi=2 \pi \alpha_{0} r_{\mathrm{ms}}^{2} n\left(r_{\mathrm{ms}}, \Theta_{\mathrm{ms}}\right) u_{0} \cos \Theta_{\mathrm{ms}} \mathrm{d} \Theta_{\mathrm{ms}}$.

Next, we assume that the sound velocity is constant at the surface $r=r_{\mathrm{ms}}$; this yields the fourth boundary condition

$c_{\mathrm{s}}\left(r_{\mathrm{ms}}, \Theta\right)=c_{0}$.

In the case of the polytropic equation of state this means that both the temperature and the relativistic enthalpy are also constant at the surface $r=r_{\mathrm{ms}}$, i.e.:

$T\left(r_{\mathrm{ms}}, \Theta\right)=T_{0}$,

$\mu\left(r_{\mathrm{ms}}, \Theta\right)=\mu_{0}$.

Therefore boundary conditions Eqs. (23)-(25) and (27) directly determine the invariants $E\left(\Theta_{\mathrm{ms}}\right)$ and $L\left(\Theta_{\mathrm{ms}}\right)$,

$E\left(\Theta_{\mathrm{ms}}\right)=\mu_{0} e_{0}$,

$L\left(\Theta_{\mathrm{ms}}\right)=\mu_{0} l_{0} \cos \Theta_{\mathrm{ms}}$,

where $e_{0}=\alpha_{0} \gamma_{0}=\sqrt{8 / 9}$ and $l_{0}=u_{\hat{\varphi}}\left(r_{\mathrm{ms}}\right) r_{\mathrm{ms}}=\sqrt{3} r_{\mathrm{g}}=2 \sqrt{3}$ (see footnote 4 ).

Condition $E=$ const. (30) allows us to rewrite the Grad-Shafranov equation in an even simpler form,

$$
\begin{aligned}
\frac{\partial^{2} \Phi}{\partial r^{2}}+\frac{\cos \Theta}{\alpha^{2} r^{2}} \frac{\partial}{\partial \Theta}\left(\frac{1}{\cos \Theta} \frac{\partial \Phi}{\partial \Theta}\right)= & -4 \pi^{2} n^{2} \frac{L}{\mu^{2}} \frac{\mathrm{d} L}{\mathrm{~d} \Phi} \\
& -4 \pi^{2} n^{2} r^{2} \cos ^{2} \Theta \frac{T}{\mu} \frac{\mathrm{d} S}{\mathrm{~d} \Phi}
\end{aligned}
$$

At $r=r_{\mathrm{ms}}$ the r.h.s. of Eq. (32) can be shown to describe the transversal balance of the pressure force and the effective potential, whereas the 1.h.s. corresponds to the dynamical term $(\boldsymbol{v} \nabla) \boldsymbol{v}$ which is $u_{0}^{2} / c_{0}^{2}$ times larger than each of the terms in the r.h.s. and thus may be dropped (Beskin et al. 2002). It is natural therefore to choose entropy $S(\Phi)$ from the condition of the transversal balance of forces at the surface $r=r_{\mathrm{ms}}$,

$r_{\mathrm{ms}}^{2} \cos ^{2} \Theta_{\mathrm{ms}} \frac{\mathrm{d} S}{\mathrm{~d} \Theta_{\mathrm{ms}}}=-\frac{\Gamma}{c_{0}^{2}} \frac{L}{\mu_{0}^{2}} \frac{\mathrm{d} L}{\mathrm{~d} \Theta_{\mathrm{ms}}}$,

where the value of $L\left(\Theta_{\mathrm{ms}}\right)$ is determined by Eq. (31). This yields the last, fifth, boundary condition

$S\left(\Theta_{\mathrm{ms}}\right)=S(0)-\frac{\Gamma}{3 c_{0}^{2}} \ln \left(\cos \Theta_{\mathrm{ms}}\right)$,

whence owing to Eq. (21) we have at $r=r_{\mathrm{ms}}$ the standard shape of concentration,

$n\left(r_{\mathrm{ms}}, \Theta\right) \approx n_{0} \exp \left(-\frac{\Gamma}{6 c_{0}^{2}} \Theta^{2}\right)$

(to be exact, $\left.n\left(r_{\mathrm{ms}}, \Theta\right)=n_{0}(\cos \Theta)^{\Gamma / 3 c_{0}^{2}}\right)$. Now with the help of Eq. (26), invariants (30), (31), and (34) can be expressed in terms of $\Phi$, therefore the Grad-Shafranov equation contains the only unknown function $\Phi(r, \Theta)$. 


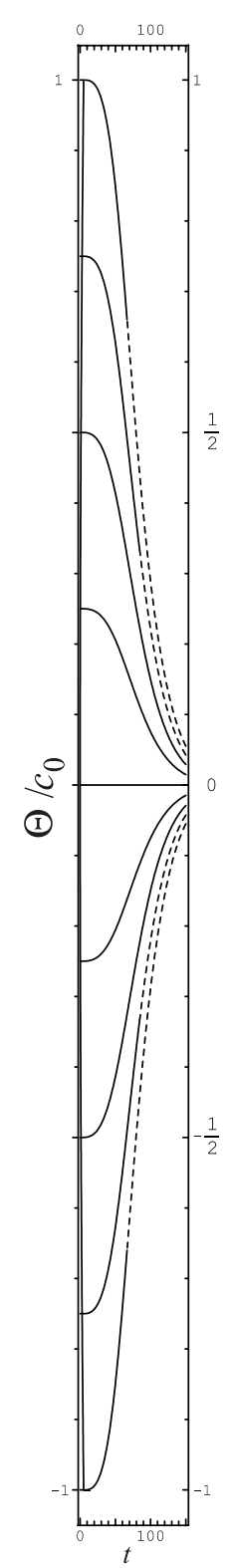

Fig. 1. The disc streamline profile (real scale) obtained from the numerical computation of Eq. (32) for $c_{0}=10^{-2}$ and $u_{0}=10^{-5}$. Solid lines correspond to the range of variables $u_{\hat{\mathrm{p}}}^{2} / c_{\mathrm{s}}^{2}<0.2$, where the solution to approximate Eq. (32) should not differ greatly from the solution to complete Eq. (14). Extrapolation of the solution to the sonic surface region is shown with dashed lines. In the figure we use the radial variable $t=\left(r_{\mathrm{ms}}-r\right) /\left(r_{\mathrm{ms}} u_{0}\right)$ : for the above choice of $u_{0}$ and $c_{0}$, the point $t=200$ corresponds to $r=2.994 r_{\mathrm{g}}$.

The disc streamline profile obtained from numerical computation of Eq. (32) is shown in Fig. 1; the profiles of radial velocity and sound speed are shown in Fig. 2. In the subsonic region, $r_{*}<r \leq r_{\mathrm{ms}} \equiv 3 r_{\mathrm{g}}$, the disc thickness rapidly diminishes, and at the sonic surface we have $H\left(r_{*}\right) \approx u_{0} / c_{0} H\left(r_{\mathrm{ms}}\right)$ so that we cannot neglect the dynamical force there (Beskin et al. 2002).

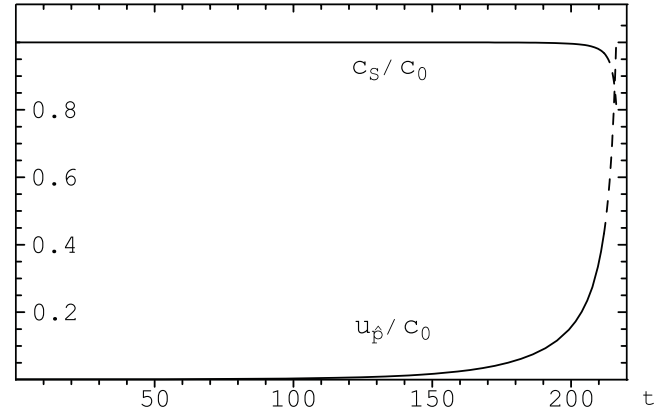

Fig. 2. Profiles of the poloidal velocity $u_{\hat{\mathrm{p}}}$ and sound velocity $c_{\mathrm{s}}$ at the equator for $c_{0}=10^{-2}$ and $u_{0}=10^{-5}$. Solid lines correspond to the same range of parameters as in Fig. 1

This can be readily derived from qualitative consideration as well. For $c_{\mathrm{s}} \ll 1$, i.e. for non-relativistic temperature, we obtain from Eqs. (18) and (20),

$u_{\hat{\mathrm{p}}}^{2}=u_{0}^{2}+w^{2}+\frac{2}{\Gamma-1}\left(c_{0}^{2}-c_{\mathrm{s}}^{2}\right)+\frac{1}{3}\left(\Theta_{\mathrm{ms}}^{2}-\Theta^{2}\right)+\ldots$

The quantity

$w^{2}(r)=\frac{e_{0}^{2}-\alpha^{2} l_{0}^{2} / r^{2}-\alpha^{2}}{\alpha^{2}} \equiv \frac{1}{\alpha^{2}} \frac{(6-r)^{3}}{9 r^{3}}$

is the poloidal four-velocity of a free particle having zero poloidal velocity at the MSO. Assuming $u_{\hat{\mathrm{p}}}=c_{\mathrm{s}}=c_{*}$ and neglecting $w^{2}$ in Eq. (37), we find the velocity of sound $c_{*}$ in the sonic point $r=r_{*}, \Theta=0$ :

$c_{*} \approx \sqrt{\frac{2}{\Gamma+1}} c_{0}$.

Since entropy $S$ remains constant along the streamlines, the gas concentration $n_{*}$ at the sonic surface slightly differs ${ }^{6}$ from the gas concentration at the MSO. In other words, the subsonic flow can be considered incompressible to a zeroth approximation $^{7}$. It is important that this conclusion holds not only in the equatorial plane because the additional term $1 / 3\left(\Theta_{\mathrm{ms}}^{2}-\Theta^{2}\right)$ in Eq. (36) is also of the order of $c_{0}^{2}$ for the range of angles corresponding to the representative disc thickness, $\Theta \lesssim \Theta_{\text {disc }} \sim c_{0}$ (see Eq. (35)). Since the density remains almost constant and the poloidal velocity increases from $u_{0}$ to $c_{*} \sim c_{0}$, i.e. changes over several orders of magnitude for $u_{0}^{2} \ll c_{0}^{2}$, the disc thickness $H$ should change in the same proportion due to the continuity equation:

$H\left(r_{*}\right) \approx \frac{u_{0}}{c_{0}} H\left(r_{\mathrm{ms}}\right)$.

Thus, a purely radial motion approximation is inapplicable in the vicinity of the sonic surface, and both components of the dynamical force become comparable to the pressure gradient near the sonic surface (Beskin et al. 2002),

$\frac{u_{\hat{\Theta}}}{r} \frac{\partial u_{\hat{\Theta}}}{\partial \Theta} \approx u_{\hat{r}} \frac{\partial u_{\hat{\Theta}}}{\partial r} \approx \frac{\nabla_{\hat{\Theta}} P}{\mu} \approx \frac{c_{0}^{2}}{u_{0}^{2}} \frac{\Theta}{r}$,

${ }^{6}$ Note that the concentration, certainly, changes from one streamline to another.

7 The same is true for spherically symmetric Bondi accretion (Shapiro \& Teukolsky 1983). 
with the position of the sonic surface $r_{*}$ being determined by

$r_{\mathrm{ms}}-r_{*}=\Lambda u_{0}^{2 / 3} r_{\mathrm{ms}}$,

where the logarithmic factor

$\Lambda=\left(\frac{3}{2}\right)^{2 / 3}\left[\ln \left(\frac{c_{0}}{u_{0}}\right)\right]^{2 / 3} \approx 5-7$,

and the radial logarithmic derivative of concentration near the sonic surface is estimated as

$\eta_{1}=(r / n)(\partial n / \partial r) \sim u_{0}^{-1}$.

In other words, if there appears a nonzero vertical velocity component, the dynamical term $(\boldsymbol{v} \nabla) \boldsymbol{v}$ cannot be neglected in the vertical force balance near the sonic surface. This property is apparently valid for arbitrary radial velocities of the flow, i.e. even if the transverse contraction of the disc is not so pronounced.

\section{Transonic flow}

In order to verify our conclusions we consider the flow structure in the vicinity of the sonic surface in more detail. Since the smooth transonic flow is analytical at a singular point $r=r_{*}, \Theta=0$ (Landau \& Lifshits 1987b), we can write

$$
\begin{aligned}
n & =n_{*}\left(1+\eta_{1} h+\frac{1}{2} \eta_{3} \Theta^{2}+\ldots\right), \\
\Theta_{\mathrm{ms}} & =a_{0}\left(\Theta+a_{1} h \Theta+\frac{1}{2} a_{2} h^{2} \Theta+\frac{1}{6} b_{0} \Theta^{3}+\ldots\right),
\end{aligned}
$$

where $h=\left(r-r_{*}\right) / r_{*}$. Here we assume that all the three invariants $E, L$, and $S$ are given by boundary conditions (30), (31), and (34) respectively. Hence, the problem needs only one more boundary condition.

The full stream Eq. (14) may be written as

$$
\begin{aligned}
& -\frac{1}{\alpha} \nabla_{k}\left(\frac{1}{\alpha \varpi^{2}} \mathcal{M}^{2} \nabla^{k} \Phi\right) \\
& +\frac{64 \pi^{4}}{\alpha^{2} \varpi^{2} \mathcal{M}^{2}}\left(\varpi^{2} E \frac{\mathrm{d} E}{\mathrm{~d} \Phi}-\alpha^{2} L \frac{\mathrm{d} L}{\mathrm{~d} \Phi}\right)-16 \pi^{3} n T \frac{\mathrm{d} S}{\mathrm{~d} \Phi}=0 .
\end{aligned}
$$

Neglecting spatial derivatives of $\mu$ here (this can be done in the vicinity of the sonic surface for $c_{0} \ll 1$ as is shown later) we get an equation with a form similar to Eq. (32), but this time the equation can be used in the vicinity of the sonic surface. Now comparing the appropriate coefficients in Bernoulli (18) and full stream Eq. (46) we obtain, neglecting terms of the order of $\varepsilon=u_{0}^{2} / c_{0}^{2}$ (see the Appendix for the exact values),

$$
\begin{aligned}
& a_{0}=\left(\frac{2}{\Gamma+1}\right)^{(\Gamma+1) / 2(\Gamma-1)} \frac{c_{0}}{u_{0}}, \\
& a_{1}=2+\frac{1-\alpha_{*}^{2}}{2 \alpha_{*}^{2}} \approx 2.25, \\
& a_{2}=-(\Gamma+1) \eta_{1}^{2}, \\
& b_{0}=\left(\frac{\Gamma+1}{6}\right) \frac{a_{0}^{2}}{c_{0}^{2}}, \\
& \eta_{3}=-\frac{2}{3}(\Gamma+1) \eta_{1}^{2}-\left(\frac{\Gamma-1}{3}\right) \frac{a_{0}^{2}}{c_{0}^{2}},
\end{aligned}
$$

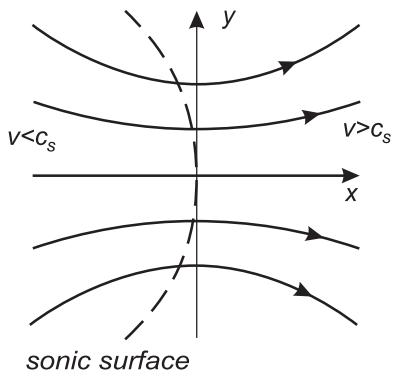

Fig. 3. Schematics of thin disc streamlines profile around the sonic point. The flow has the form of a standard nozzle. Here $x=-h$, and $y=\Theta$.

where $\alpha_{*}^{2}=\alpha^{2}\left(r_{*}\right) \approx 2 / 3$. As we see, coefficients (47)-(51) are expressed through the radial logarithmic derivative $\eta_{1}$ (which we specify according to Eq. (43) as the fourth boundary condition). They have clear physical meanings. So, $a_{0}$ gives the compression of streamlines: $a_{0}=H\left(r_{\mathrm{ms}}\right) / H\left(r_{*}\right)$. In agreement with Eq. (39) we have $a_{0} \approx c_{0} / u_{0}$. Further, $a_{1}$ corresponds to the slope of the streamlines with respect to the equatorial plane. As $a_{1}>0$, the compression of the streamlines finishes somewhere before the sonic surface, so inside the sonic radius $r<r_{*}$ the streamlines diverge. On the other hand, because $a_{1} \ll u_{0}^{-1}$, for $r=r_{*}$ the divergency is still very weak. Hence in the vicinity of the sonic surface the flow has the form of the standard nozzle (see Fig. 3). Finally, since $a_{2} \sim \eta_{3} \sim b_{0} \sim u_{0}^{-2}$, the transverse scale of the transonic region $H\left(r_{*}\right)$ is the same as the longitudinal one. This means that the transonic region is essentially two-dimensional (a well-known fact for a nozzle, see e.g. Landau \& Lifshits 1987b), and it is impossible to analyze it within the standard one-dimensional approximation.

Since the transonic flow in the form of a nozzle has longitudinal and transversal scales of one order of magnitude, near the sonic surface we have $\delta r_{\|} \approx \delta r_{\perp}$, i.e.

$\delta r_{\|} \approx H\left(r_{*}\right)$.

Hence, for thin discs this longitudinal scale is always much smaller than the distance from the $\mathrm{BH}, \delta r_{\|} / r_{*} \approx H\left(r_{*}\right) / r_{*} \ll$ 1. Only by taking the transversal velocity into account do we retain the small longitudinal scale $\delta r_{\|} \ll r_{\mathrm{g}}$. This scale is left out during the standard one-dimensional approach.

We stress that taking the dynamical force into account is extremely important. This is because, unlike the zero-order standard disc thickness prescription (1), the Grad-Shafranov equation has second order derivatives, i.e. contains two additional degrees of freedom. This means that the critical condition only fixes one of these degrees of freedom (e.g. imposes some limitations on the form of the flow) rather than determines the angular momentum of the accreting matter.

Connecting the sonic characteristics $\eta_{1}=\eta_{1}\left(r_{*}\right)$ with the physical boundary conditions on the MSO $r=r_{\mathrm{ms}}$ is rather difficult (for this we have to know all the expansion coefficients in Eqs. (44) and (45)). In particular, we cannot formulate the restriction on five boundary conditions (23)-(25), (27), and (34) resulting from the critical condition on the sonic surface. Nevertheless, estimate (43) of $\eta_{1}$ makes sure that we know 
the parameter $\eta_{1}$ to a high enough accuracy. Then, according to Eqs. (47)-(51), all other coefficients can be determined exactly.

Using expansions (44) and (45), one can obtain all other physical parameters of the transonic flow. In particular, we have

$$
\begin{aligned}
u_{\hat{\mathrm{p}}}^{2}= & c_{*}^{2}\left[1-2 \eta_{1} h+\frac{1}{6}(\Gamma-1) \frac{a_{0}^{2}}{c_{0}^{2}} \Theta^{2}+\frac{2}{3}(\Gamma+1) \eta_{1}^{2} \Theta^{2}\right], \\
c_{\mathrm{s}}^{2}= & c_{*}^{2}\left[1+(\Gamma-1) \eta_{1} h+\frac{1}{6}(\Gamma-1) \frac{a_{0}^{2}}{c_{0}^{2}} \Theta^{2}\right. \\
& \left.-\frac{1}{3}(\Gamma-1)(\Gamma+1) \eta_{1}^{2} \Theta^{2}\right] .
\end{aligned}
$$

Therefore, the sonic surface, $u_{\hat{\mathrm{p}}}=c_{\mathrm{s}}$, has the standard parabolic form:

$h=\frac{1}{3}(\Gamma+1) \eta_{1} \Theta^{2}$.

Finally, Eq. (53) enables us to estimate the spatial derivatives of $\mu$. Then, it can be easily shown that the terms containing these derivatives in Eq. (46) are negligible for $c_{0} \ll 1$ and indeed can be safely dropped. Therefore the validity of neglecting the derivatives of $\mu$ in Eq. (46)has been justified.

\section{Supersonic flow}

Since the pressure gradient becomes insignificant in the supersonic region, the matter here moves along the trajectories of free particles. Neglecting the $\nabla_{\theta} P$ term in the $\theta$-component of relativistic Euler equation (Frolov \& Novikov 1998), we have (compare to Abramowicz et al. 1997)

$\alpha u_{\hat{r}} \frac{\partial\left(r u_{\hat{\Theta}}\right)}{\partial r}+\frac{\left(r u_{\hat{\Theta}}\right)}{r^{2}} \frac{\partial\left(r u_{\hat{\Theta}}\right)}{\partial \Theta}+\left(u_{\hat{\varphi}}\right)^{2} \tan \Theta=0$.

Here, using the conservation law of angular momentum, $u_{\hat{\varphi}}$ can be easily expressed in terms of radius: $u_{\hat{\varphi}}=2 \sqrt{3} / r$. We also introduce the dimensionless functions $f(r)$ and $g(r)$ :

$$
\begin{aligned}
\Theta f(r) & =r u_{\hat{\Theta}}, \\
g(r) & =-\alpha u_{\hat{r}}>0 .
\end{aligned}
$$

Using Eq. (55) and the definitions above, we obtain an ordinary differential equation for $f(r)$ which could be solved if we knew $g(r)$ :

$$
\frac{\mathrm{d} f}{\mathrm{~d} r}=\frac{f^{2}+12}{r^{2} g(r)} \text {. }
$$

From Eq. (18) we have $u_{\hat{\mathrm{p}}}^{2} \rightarrow w^{2}$ as $r \rightarrow r_{\mathrm{g}}$. On the other hand, $u_{\hat{\mathrm{p}}} \approx c_{*} \approx c_{0}$ for $r \lesssim r_{*}$. Therefore, the following approximation should be valid throughout the $r_{\mathrm{g}}<r<r_{*}$ region,

$g(r) \approx \sqrt{(\alpha w)^{2}+\left(\alpha c_{*}\right)^{2}}$.

Equation (58) governs the supersonic flow structure for the case of a non-spinning $\mathrm{BH}$. To get a better match with observations (cf. Sect. 6), we also consider a more general case of a spinning BH, i.e. a Kerr BH with non-zero specific angular momentum $a$. After some calculations, Eq. (58) can be generalized to the Kerr metric with a strikingly simple form,

$\frac{\mathrm{d} f}{\mathrm{~d} r}=\frac{f^{2}+a^{2}\left(1-e_{0}^{2}\right)+l_{0}^{2}}{r^{2} \tilde{g}(r)}$.

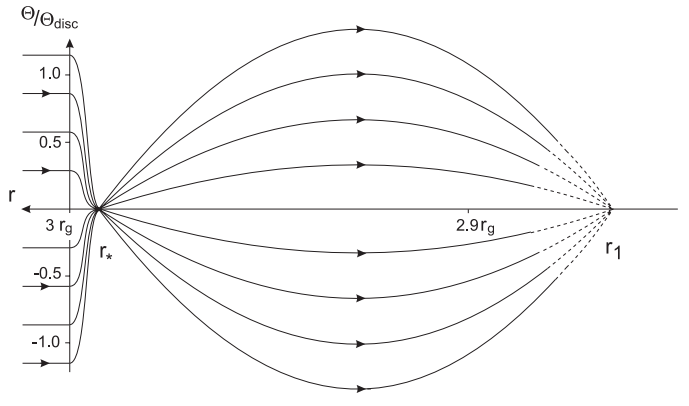

Fig. 4. The structure of a thin accretion disc (actual scale) for $c_{0}=$ $10^{-2}, u_{0}=10^{-5}$ after passing the MSO $r=3 r_{\mathrm{g}}$ (Schwarzschild case). As sufficient dissipation can take place in the vicinity of the first node $r=r_{1}$, we do not prolong the flow lines to the region $r<r_{1}$.

Here $e_{0}$ and $l_{0}$ are the specific energy and the angular momentum of a free particle rotating at the MSO respectively (see e.g. Shapiro \& Teukolsky 1983), and $\tilde{g}(r)$ is a straightforward generalization of $g(r)$ to the Kerr case. For the Schwarzschild $\mathrm{BH}\left(a=0, e_{0}=\sqrt{8 / 9}, l_{0}=2 \sqrt{3}\right)$ Eq. (60) reduces back to Eq. (58).

Integrating Eq. (60), we obtain

$f(r)=\kappa \tan \left[\kappa \int_{r_{*}}^{r} \frac{\mathrm{d} \xi}{\xi^{2} g(\xi)}+\frac{\pi}{2}\right]$

where $\kappa=\sqrt{a^{2}\left(1-e_{0}^{2}\right)+l_{0}^{2}}$. In the equation above, $\pi / 2$ has been to a good accuracy substituted for the integration constant $\arctan \left[f\left(r_{*}\right) / \sqrt{\kappa}\right]$ : for $r$ just below $r_{*}$, the function $f$ is positive reflecting the fact that the flow diverges; then, $f=0$ corresponds to the point where the divergence finishes, and the flow starts to converge. The results of numerical calculations are presented in Fig. 4. In the supersonic region the flow performs transversal oscillations about the equatorial plane, their frequency independent of their amplitude. We see as well that the maximum thickness of the disc in the supersonic (and, hence, ballistic) region, which is controlled by the transverse component of the gravitational force, actually coincides with the disc thickness within the stable orbits region, $r>r_{\mathrm{ms}}$, where standard estimate (1) is correct.

Once diverged, the flow converges once again at a "nodal" point closer to the $\mathrm{BH}$. The radial positions of the nodes are given by the implicit formula $f\left(r_{n}\right)= \pm \infty$, i.e.

$\kappa \int_{r_{n}}^{r_{*}} \frac{\mathrm{d} \xi}{\xi^{2} g(\xi)}=n \pi$

where $n=0,1,2, \ldots$ is the node number; the node with $n=0$ corresponds to the sonic surface. In this formula the sonic radius $r_{*} \equiv r_{0}$ can be to a good accuracy approximated by $r_{\mathrm{ms}}=r_{\mathrm{ms}}(a)$ for $u_{0} \ll c_{0} \ll 1$ (see Eq. (41)). The expression for $r_{\mathrm{ms}}(a)$ can be found in most textbooks (Shapiro \& Teukolsky 1983).

Note that Eqs. (58) and (60) are inapplicable in the vicinity of the nodes where the pressure gradient cannot be neglected. There may also be an additional energy release because the shock fronts (and, hence, extra sonic surfaces) inevitably appear there. These factors can reduce the amplitude of the disc thickness oscillations. Accurate analysis of these factors lies 


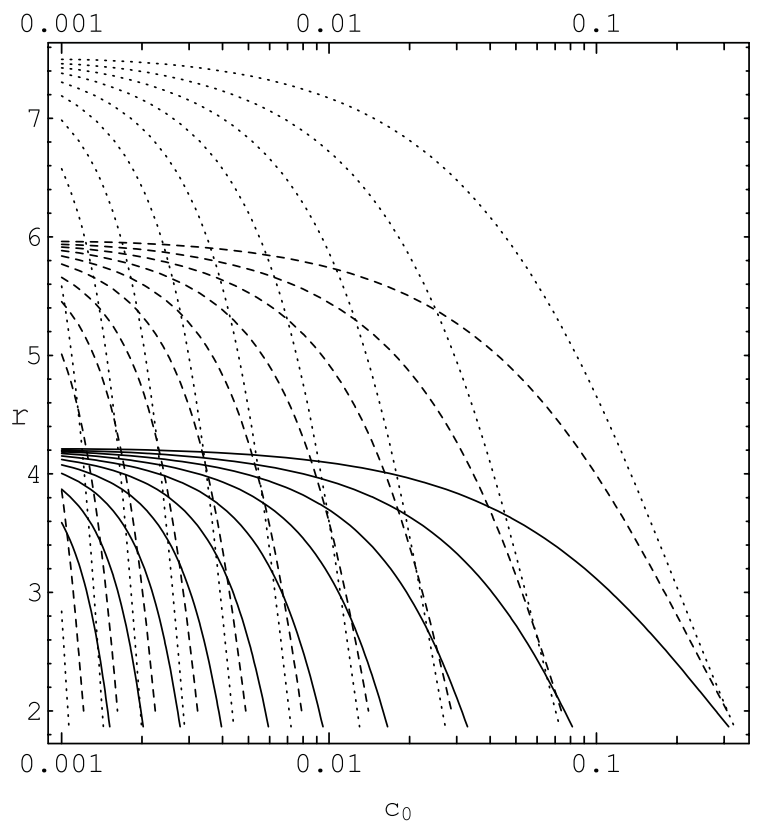

Fig. 5. Radial positions (in the units of $M$ ) of the nodes for a range of initial sound velocities. Dotted, dashed, and solid curves correspond to the cases $a=-0.5, a=0$, and $a=0.5$ respectively. Each curve relates the radial position of a node to a value of the initial sound velocity. Intersection points of these curves with the line $c_{0}=$ const give the the nodes' radial positions for that particular value of $c_{0}$.

beyond the scope of this paper, and we do not prolong the streamlines to the region $r<r_{1}$ in Fig. 4. Nevertheless, it is natural to suppose that the nodes positions are not significantly affected by the dissipative processes, and our simple approach may give us a good qualitative understanding of the supersonic flow structure as long as we are interested in general properties of the flow.

Figure 5 shows the positions of nodes for different values of $c_{0}$ (the positions do not depend on $u_{0}$ for $u_{0} \ll c_{0}$ ) and the BH spin parameter $a$.

Travel time between the nodes, which is similar to the halfperiod of vertical epicyclic oscillations, proves to have a weak dependence on both $u_{0}$ and $c_{0}$. This fact provides a means for testing the theory via observations, and we do this in the following section.

\section{Applications to observations}

\subsection{General ideas}

Our initial interest in our model's application to observations was kindled by the publication by Genzel et al. (2003) of the infrared observations of the Galactic Centre (GC): one of the flares obviously showed a number of peaks at time intervals decreasing with time (see their Fig. 2e), and this is exactly what we would expect within our model. In this section we measure physical radial distances in the units of $G M / c^{2}$ and time intervals in the units of $G M / c^{3}$.
Suppose some perturbation in the disc (a "chunk") approaches the MSO. We expect to observe radiation coming from the chunk with the period of its orbital motion,

$T_{\mathrm{ms}}(a)=2 \pi\left(r_{\mathrm{ms}}^{3 / 2}+a\right)$

where $a$ is the angular momentum per unit mass of the $\mathrm{BH}$ and $r_{\mathrm{ms}}$ is the estimate of the distance from the $\mathrm{BH}$ to the chunk. After a number of rotations, the chunk reaches the MSO and passes through the nodal structure derived earlier (cf. Sect. 5) generating a flare. Each time the chunk passes through a node, it probably generates some additional radiation, and therefore the flare is likely to consist of several peaks. We believe that it is these peaks that were discovered in the infrared and X-ray observations of the GC (Genzel et al. 2003; Aschenbach et al. 2004).

We stress that this section is independent of other parts of this work. Qualitatively, our model's only assumptions are the small thickness of the disc and the divergence of the flow in the supersonic region (the increase of disc thickness with decreasing radius). The latter is guaranteed provided that the flow has a nozzle-like structure (see Sect. 4) near the sonic point.

\subsection{Finding the periods}

The time interval between the detection of two subsequent peaks ( $n$th and $(n-1)$ th) equals the time it takes for the chunk to pass between the two corresponding adjacent nodes ( $n$th and $(n-1)$ th), $T_{n}^{(1)}$, plus the difference in travel times to the observer for the radiation coming from these two nodes, $T_{n}^{(2)}$ :

$T_{n}=T_{n}^{(1)}+T_{n}^{(2)}$

$n$ can also be thought of as the index of observed time intervals (counting from one).

The last term in Eq. (64) is important when one of the two nodes comes close to the BH horizon: in such a case time dilation makes it significantly longer for the radiation to reach the observer from that node which significantly increases $T_{n}^{(2)}$. However, for outer nodes this can never happen. With this in hand, we can estimate the value of $T_{1}$ neglecting the effect of $T_{1}^{(2)}$. In the supersonic region, the particles move along almost ballistic trajectories, and the orbit of a particle falling off the MSO is the circle inclined with respect to the equatorial plane (so that the centre of the orbit is the singularity of the $\mathrm{BH}$ ) and perturbed by the slow radial inflow motion. This is especially true of outermost nodes where the matter has moderate radial velocities and time dilation is negligible.

Suppose that at some moment in time the particle is located at the sonic point (on the equatorial plane) which is very close to the MSO $r=r_{\mathrm{ms}}$ (see Eq. (41)). Having travelled for half the orbital period, the particle would have reached the equatorial plane again, i.e. the first node, therefore

$T_{1} \lesssim 1 / 2 T_{\mathrm{ms}}(a)$.

This shows that the first time interval is a little shorter than half the orbital period at the MSO. Therefore, we would expect the observations to exhibit not only the rotational frequency at the 


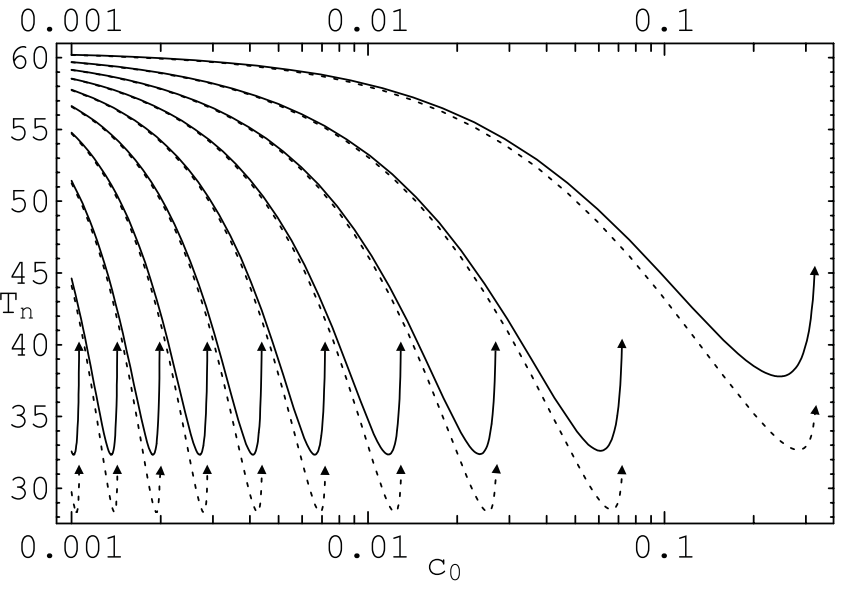

Fig. 6. The dependence of time intervals between the peaks in a flare on the speed of sound in the disc, $c_{0}$, for a moderately spinning $\mathrm{BH}$ ( $a=-0.5$ ); we measure time intervals in the units of $G M / c^{3}$. Solid curves correspond to $T_{n}=T_{n}^{(1)}+T_{n}^{(2)}$ whereas dashed ones correspond to $T_{n}^{(1)}$. The uppermost solid curve corresponds to the time interval $T_{1}$ between the 0th and 1st peaks, the second solid curve from the top corresponds to the time interval $T_{2}$ between the 1 st and 2 nd ones, etc. Even though individual time intervals between subsequent peaks in the flare may depend on the temperature in the disc (which is proportional to $c_{0}^{2}$, see Eq. (20)), their minimum and maximum values remain the same for the range of sound velocities where there are several intervals observed.In the particular case of $a=-0.5$, illustrated in the figure, we have $T_{\min } \approx 32$ and $T_{\max } \approx 60$ with all other time intervals lying in between. For $M=3.7 \times 10^{6} M_{\odot}$ we obtain $T_{\min } \approx 600 \mathrm{~s}$ and $T_{\max } \approx 1100 \mathrm{~s}$.

MSO but also its double counterpart, i.e. the frequency close to twice the rotational frequency.

Accurate calculations of $T_{n}^{(1)}-$ the observer's time it takes for the matter to travel from the $(n-1)$ th node to the $n$th one yield

$T_{n}^{(1)}\left(a, c_{\mathrm{s}}\right)=\int_{r_{n}}^{r_{n-1}} \frac{u^{t}}{u^{r}} \mathrm{~d} r=\int_{r_{n}}^{r_{n-1}} \frac{\left.\left(-e_{0} g^{t t}+l_{0} g^{t \varphi}\right)\right|_{\theta=\pi / 2}}{g(r)} \mathrm{d} r$,

where the coefficients of the inverse metric are $g^{t t}=-\Sigma^{2} /\left(\rho^{2} \Delta\right)$ and $g^{t \varphi}=\omega g^{t t}$; the definitions of $\Sigma, \rho, \Delta$, and $\omega$ can be found in Thorne et al. (1986). The resulting relationship $T_{n}^{(1)}\left(a, c_{0}\right)$ for $a=-0.5$ is shown in Fig. 6 with dashed lines.

For definiteness and simplicity, we assume that the observer is located along the rotation axis of the $\mathrm{BH}$. On its way to the observer, the radiation travels along the null geodesic that originates at a node in the equatorial plane (e.g. $r=r_{n}, \theta=\pi / 2$ ) and reaches the observer at infinity $(r=\infty, \theta=0)$. Using these as boundary conditions for null geodesics in the Kerr metric (Carter 1968), we numerically find $T_{n}^{(2)}\left(a, c_{0}\right)$.

Figure 6 shows the dependence of observed time intervals on the value of the speed of sound in the disc. All intervals $T_{n}$ behave very similarly: they first decrease with increasing $c_{0}$ and then abruptly increase to infinity due to time dilation. This increase occurs if the innermost node in the pair of nodes comes close to the BH horizon (this is indicated with upward arrows). Although each individual time interval may depend on $c_{0}$, the range $\left[T_{\min }(a), T_{\max }(a)\right]$ of observed time intervals (see the caption to Fig. 6) is independent of $c_{0}$ for those $c_{0}$ where

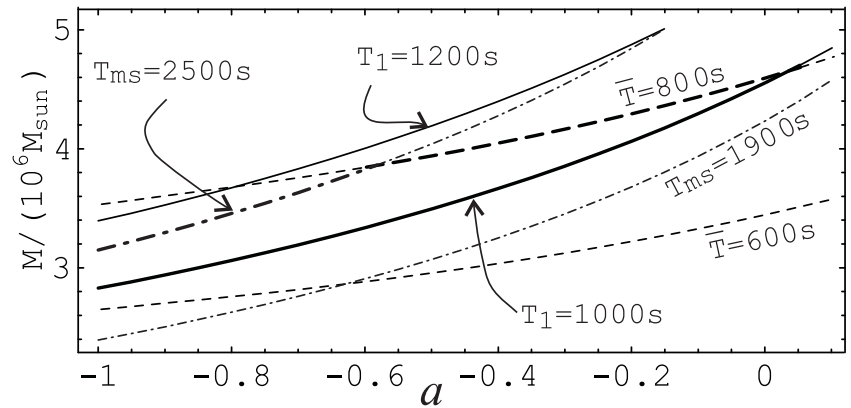

Fig. 7. Relation of $M$ vs. $a$. Dashed, dash-dotted, and solid lines come from matching $\bar{T}(700 \pm 100 \mathrm{~s}), T_{\mathrm{ms}}(2200 \pm 300 \mathrm{~s})$, and $T_{0}(1100 \pm 100 \mathrm{~s})$ respectively. The resulting error polygon is bolded.

there are several peaks observed. With such weak dependence on the speed of sound in the disc, we have only two matching parameters: the specific spin $a$ and the mass $M$ of the $\mathrm{BH}$.

\subsection{Matching the observations}

In the flare precursor section we associate the period $T_{\mathrm{ms}}$ with the $2200 \pm 300$ s one (group 5, cf. Table 2 in Aschenbach et al. 2004 ) and the time interval $T_{1}$ with the period of $1100 \pm 100 \mathrm{~s}$ (group 4 in Aschenbach et al. 2004). Consistent with the infrared observations of the flare, the time intervals $T_{1}, T_{2}$, etc. decrease with the peak number (Genzel et al. 2003), i.e. resemble the QPO structure and thus form a cumulative peak of a larger width shifted to higher frequencies on the flares' power density spectra $(700 \pm 100 \mathrm{~s}$, group 3 , cf. Figs. 3a and 4a in Aschenbach et al. 2004). We can estimate the average frequency of this peak as $1 / \bar{T}=1 / 2\left(1 / T_{\min }+1 / T_{\max }\right)$.

The results of the periods' matching procedure are shown in Fig. 7. Observational data clearly rules out high positive values of $a$ (i.e. the disc orbiting in the same direction as the BH spin), and therefore the accretion disc around the Central Black Hole is likely to be counter-rotating. Moreover, the data presented in Fig. 7 enable us to estimate the mass of the Central Black Hole as $(3.7 \pm 1) \times 10^{6} M_{\odot}$. This value is in agreement with the studies by Schödel et al. (2002) and Chez et al. (2003).

\section{Conclusions}

Allowing for the vertical velocity in the vertical force balance, i.e. performing the two-dimensional analysis, is extremely important, especially near the sonic surface and in the supersonic region. Two-dimensional analysis reveals the existence of a new small radial scale, which is of the order of the thickness of the disc, near the sonic surface. It also shows that the critical condition fixes neither the accretion rate nor the angular momentum of the infalling matter. The nozzle-like shape of the flow around the sonic point leads to the oscillations of disc thickness in the supersonic region that enable us to propose a straightforward interpretation of quasi-periodical oscillations observed in the radiation coming from the GC (Genzel et al. 2003; Aschenbach et al. 2004). This constrains the mass of the Central Black Hole to $(3.7 \pm 1) \times 10^{6} M_{\odot}$ which is in agreement with other studies (Schödel et al. 2002; Chez et al. 2003). Our 
interpretation also suggests that the accretion disc around the Galactic Centre black hole is counter-rotating.

Acknowledgements. We thank A. V. Gurevich for his interest in the work and for his support, useful discussions and encouragement. We are very grateful to K. A. Postnov for his help and fruitful suggestions regarding the observational part. A.D.T. thanks T. Elmgren for making valuable corrections to the text. This work was supported by the Russian Foundation for Basic Research (grant No. 1603.2003.2), Dynasty fund, and ICFPM.

\section{Appendix A: Exact values of expansion coefficients}

In this section we provide exact values of the expansion coefficients (47)-(51). Here we account for the derivatives of the metrics and keep terms of higher orders of smallness:

$$
\begin{aligned}
a_{0}= & \frac{\alpha_{*} r_{*}^{2}}{\alpha_{0} r_{\mathrm{ms}}^{2}}\left(\frac{2}{\Gamma+1}\right)^{(\Gamma+1) / 2(\Gamma-1)} \frac{c_{0}}{u_{0}}, \\
a_{1}= & +\frac{1-\alpha_{*}^{2}}{2 \alpha_{*}^{2}}, \\
a_{2}= & 6 a_{1}-6-a_{1}^{2}-(\Gamma+1) \eta_{1}^{2}, \\
b_{0}= & -1-\alpha_{*}^{2} a_{1}^{2}+\left(\frac{\Gamma+1}{6}\right) \frac{a_{0}^{2}}{c_{0}^{2}}, \\
\eta_{3}= & \frac{2}{3}\left(6 a_{1}-6-a_{1}^{2}-\eta_{1} a_{1}-\frac{3}{2} \alpha_{*}^{2} a_{1}^{2}\right) \\
& \quad-\frac{\Gamma+1}{6} \frac{a_{0}^{2}-1}{c_{0}^{2}}-\frac{\Gamma}{3} \frac{a_{0}^{2}}{c_{0}^{2}}-\frac{\Gamma+1}{6}\left(1-\frac{c_{*}^{2} r_{*}^{2}}{c_{0}^{2} r_{\mathrm{ms}}^{2}}\right) \frac{r_{*}^{2}}{r_{\mathrm{ms}}^{2}} \frac{a_{0}^{2}}{c_{0}^{2}} \\
& \quad-\frac{2}{3}(\Gamma+1) \eta_{1}^{2},
\end{aligned}
$$

where $\alpha_{*}=\alpha\left(r_{*}\right)$ and $\alpha_{0}=\alpha\left(r_{\mathrm{ms}}\right)$.

\section{References}

Abramowicz, M. A., \& Zurek, W. 1981, ApJ, 246, 314

Abramowicz, M. A., Lanza, A., \& Percival, M. J. 1997, ApJ, 479, 179

Abramowicz, M. A., Czerny, B., Lasota, J.-P., \& Szuszkiewicz, E. 1988, ApJ, 332, 646

Artemova, Yu. V., Bisnovatyi-Kogan, G. S., Igumenshchev, I. V., \& Novikov, I. D. 2001, ApJ, 549, 1050
Aschenbach, B., Grosso, N., Porquet, D., et al. 2004, A\&A, 417, 71

Balbus, S. A., \& Hawley, J. F. 1998, Rev. Mod. Phys., 70, 1

Beloborodov, A. M. 1998, MNRAS, 297, 739

Beskin, V. S. 1997, Phys. Usp., 40, 659

Beskin, V. S., \& Pariev, V. I. 1993, Phys. Usp., 36, 529

Beskin, V. S., Kompaneetz, R. Yu., \& Tchekhovskoy, A. D. 2002, Astron. Lett., 28, 543

Bondi, H. 1952, MNRAS, 112, 195

Carter, B. 1968, Phys. Rev., 174, 5

Chakrabarti, S. 1996, ApJ, 471, 237

Chen, X., Abramowicz, M. A., \& Lasota, J.-P. 1997, ApJ, 476, 61

Chez, A. M., Duchêne, G., Matthews, K., et al. 2003, ApJ, 586, L127

De Villers, J., Hawley, J. F., \& Krolik, J. H. 2003, ApJ, 599, 1238

Frolov, V. P., \& Novikov, I. D. 1998, Black Hole Physics (Dordrecht: Kluwer Academic Publishers)

Gammie, C. F., \& Popham, R. 1998a, ApJ, 498, 313

Gammie, C. F., \& Popham, R. 1998b, ApJ, 504, 419

Gammie, C. F., Shapiro, S. L., \& McKinney, J. C. 2004, ApJ, 602, 312

Genzel, R., Schödel, R., Ott, T., et al. 2003, Nature, 425, 934

Igumenshchev, I. V., \& Beloborodov, A. M. 1997, MNRAS, 284, 767

Igumenshchev, I. V., Abramowicz, M. A., \& Narayan, R. 2000, ApJ, 537, L27

Igumenshchev, I. V., Narayan, R., \& Abramowicz, M. A. 2003, ApJ, 592,1042

Krolik, J. H., \& Hawley, J. F. 2002, ApJ, 573, 754

Landau, L. D., \& Lifshits, E. M. 1987a, The Classical Theory of Fields, 4th edn. (Butterworth-Heinemann)

Landau, L. D., \& Lifshits, E. M., 1987b, Fluid mechanics, 2nd edn. (Butterworth-Heinemann)

Lipunov, V. M. 1992, Astrophysics of Neutron Stars (Berlin: SpringerVerlag)

Lynden-Bell, D. 1969, Nature, 223, 690

Narayan, R., Kato, S., \& Honma, F. 1997, ApJ, 476, 49

Novikov, I. D., \& Thorne, K. S., 1973, in Black Holes, ed. C. DeWitt, B. DeWitt (New York: Gordon and Breach)

Paczyński, B., \& Bisnovatyi-Kogan, G. S. 1981, Acta Astron., 31, 283

Paczyński, B., \& Wiita, P. J. 1980, A\&A, 88, 23

Papaloizou, J., \& Szuszkiewicz, E. 1994, MNRAS, 268, 29

Peitz, J., \& Appl, S. 1997, MNRAS, 286, 681

Riffert, H., \& Herold, H. 1995, ApJ, 450, 508

Schödel, R., Ott, T., Genzel, R., et al. 2002, Nature, 419, 664

Shakura, N. I. 1972, AZh, 49, 921

Shakura, N. I., \& Sunyaev, R. A. 1973, A\&A, 24, 337

Shapiro, S. L., \& Teukolsky, S. A. 1983, Black Holes, White Dwarfs, and Neutron Stars (New York: Wiley-Interscience Publication)

Thorne, K. S., Price R. N., \& Macdonald, D. A. 1986, Black Holes: The Membrane Paradigm (New Haven, CT: Yale Univ. Press) 